\title{
Mathematical Models of Physiological Responses to Exercise
}

\author{
Somayeh Sojoudi, Benjamin Recht, and John C. Doyle
}

\begin{abstract}
This paper develops empirical mathematical models for physiological responses to exercise. We first find single-input single-output models describing heart rate variability, ventilation, oxygen consumption and carbon dioxide production in response to workload changes and then identify a single-input multi-output model from workload to these physiological variabilities. We also investigate the possibility of the existence of a universal model for physiological variability in different individuals during treadmill running. Simulations based on real data substantiate that the obtained models accurately capture the physiological responses to workload variations. In particular, it is observed that (i) different physiological responses to exercise can be captured by low-order linear or mildly nonlinear models; and (ii) there may exist a universal model for oxygen consumption that works for different individuals.
\end{abstract}

\section{INTRODUCTION}

The area of system identification deals with building mathematical models for physical processes based on experimental measurements. Several methods and algorithms have been developed in the literature for the identification of a linear timeinvariant (LTI) system in both time and frequency domains [1]-[4]. However, nonlinear system identification is a more difficult problem and there are many challenging issues that are yet unsolved [5], [6]. If the structure of the nonlinear system to be identified is known a priori, the identification process amounts to a parameter estimation problem. The main difficulty associated with a nonlinear parameter estimation problem is the inability to find a globally optimal solution in general. It is often the case that no prior information is available about the structure of the system being identified. To address this problem, different partially-successful methods have been proposed in the literature, which are based on Volterra series, Wiener series, or basis function representation of an approximate model of the system [7]-[9].

The Hammerstein system is a cascade connection of a static nonlinearity followed by a linear dynamic system. Hammerstein system identification has become popular due to its wide range of applications [9]-[11]. Several identification approaches have been proposed for these systems, such as least squares [9], subspace approach [12], and Kernel-based approach [13].

It is known that every LTI discrete-time system with fading memory can be approximated arbitrarily precisely by an LTI model followed by a static nonlinear part. Such a nonlinear

Email: sojoudi@berkeley.edu,_brecht@berkeley.edu, and doyle@ caltech.edu

Somayeh Sojoudi is with the Departments of Electrical Engineering and Computer Sciences and Mechanical Engineering, University of California, Berkeley. Benjamin Recht is with the Department of Electrical Engineering and Computer Sciences, University of California, Berkeley. John C. Doyle is with the Department of Control and Dynamical systems, California Institute of Technology. This work was in part supported by the ONR Award N0001418-1-2526 and NSF Award 1808859 system is referred to as Wiener system. This implies that although the space of nonlinear fading-memory systems is utterly complex, the nicely characterizable set of Wiener systems is dense in this space [14]. Although the class of Hammerstein systems is a counterpart for the class of Weiner systems, no result has been reported in the literature to date about the denseness of this class in the space of nonlinear fading-memory systems.

Several system identification methods have been extensively used to find biological models $[15]-[19]$. The identification of physiological variability during exercise is an important problem whose goal is to obtain simple physiological models. Such models can be used for a wide variety of biomedical applications, such as the prediction of the most appropriate changes in treatment, diet, or exercise program. In particular, the model identification of heart rate variability and oxygen consumption during exercise has been the center of attention in several researches [20]- [21]. For instance, the paper [20] models the heart rate response during and after treadmill walking exercise as a feedback interconnected nonlinear system. The work [22] also studies the feasibility of automatic feedback control of oxygen uptake during treadmill exercise, which requires the parameter identification of both the plant and the feedback controller. By combining system identification with basic physiological models, paper [23] shows that robust efficiency and actuator saturation tradeoffs can explain healthy human heart rate variability.

The primary objective of this work is to find an appropriate model for the physiological variability in response to exercise. We aim to empirically show that most of the physiological variabilities can be modeled by low-order linear or mildly nonlinear (Hammerstein) dynamical systems. To this end, we use the existing modeling techniques in system identification, but develop customized local search algorithms to solve such nonconvex estimation problems (since we have observed that an off-the-shelf algorithm would result in a local solution whose corresponding model does not fit our data well). More precisely, one major challenge with the existing system identification techniques (including those surveyed above) is that they often require access to a global optimization solver to find the best model. As a remedy, practitioners often use various local search algorithms and reformulation techniques to solve those estimation problems, but then the accompanying theoretical guarantees on system identification are no longer valid. The issue of dealing with nonconvexity for data-driven problems exists in machine learning as well, and there is a recent line of research that shows certain classes of learning problems do not have spurious local minima and therefore customized local search algorithms (with saddle-point escaping properties) could then find a global solution [24]-[28]. Inspired by this, we develop customized numerical algorithms 
to learn the underlying physiological models, which are based on iterative techniques and could in principle converge to a sub-optimal solution that is a non-global local solution or a saddle point. However, our simulations on real data confirm that these algorithms indeed converge to satisfactory solutions. This could, in part, be justified by the abovementioned results in machine learning and the findings of the recent paper [29] stating that gradient descent efficiently converges to a global optimizer of the maximum likelihood objective of an unknown single-input single-output system under certain conditions.

The remainder of the paper is organized as follows. Numerical algorithms are presented in Section II. Empirical models are obtained based on real data in Section III, and several important observations are made accordingly. Concluding remarks are drawn in Section IV.

\section{CUSTOMIZED LOCAL SEARCH ALGORITHMS}

The objective of this work is to find a mathematical model describing the physiological response to different levels of exercise intensity. It is assumed that this model takes workload $(W)$ as the input and generates four outputs, namely heart rate $(H R)$, ventilation $(V E)$, oxygen $(O 2)$ and difference of carbon dioxide and oxygen (CO2-O2). In this part, we adopt the existing system identification techniques, but develop customized numerical algorithms to solve nonconvex estimation problems for finding a satisfactory model of exercise based on the data collected from biking or running on treadmill. One of the main challenges associated with the underlying problem is that the structure of the underlying system from $W$ to $H R, V E, O 2, C O 2-O 2$ is not known a priori, and indeed this system may be highly nonlinear. To simplify the problem, we first restrict our attention to the system identification of linear single-input single-output (SISO) systems, which can be used to identify the best model from $W$ to each of the outputs $H R, V E$, and $O 2$. The method will later be generalized to the system identification of nonlinear single-input multi-output (SIMO) models, as the objective of the present work demands. A secondary goal of this paper is to study the possibility of the existence of a universal model that fits the physiological variability of different individuals.

\section{A. Algorithm for linear SISO systems}

Given a positive discrete time instant $\tau$ and two sequences of scalar numbers $\hat{\mathbf{u}}:=\{\hat{u}[k]\}_{k=0}^{\tau}$ and $\hat{\mathbf{y}}:=\{\hat{y}[k]\}_{k=0}^{\tau}$, it is desired to obtain a discrete-time SISO system with the known order $n \in \mathbf{N}$ such that when the input $\hat{\mathbf{u}}$ is applied to the system, its output on the time interval $[0, \tau]$ becomes as closely to $\hat{\mathbf{y}}$ as possible. The model being sought is assumed to be of the form

$$
\begin{aligned}
& x[k+1]=A x[k]+B u[k] \\
& y[k]=C x[k]+d, \quad k=0,1,2, \ldots
\end{aligned}
$$

where $x[k] \in \Re^{n}, u[k] \in \Re$ and $y[k] \in \Re$ denote the state, input and output of the system, respectively, and $d \in \Re$ is an offset term. The unknown parameters of this system are $A$, $B, C, d$ and $x[0]$. Note that the number of scalar parameters involved in the system identification problem is equal to $(n+$ $1)^{2}+n$ in general. However, one can realize the system in a canonical form to reduce this number to $3 n+1$. More precisely, assume with no loss of generality that

$$
\begin{aligned}
A & =\left[\begin{array}{cccccc}
a_{1} & 1 & 0 & 0 & \ldots & 0 \\
a_{2} & 0 & 1 & 0 & \ldots & 0 \\
\vdots & \vdots & \vdots & \vdots & \vdots & \vdots \\
a_{n} & 0 & 0 & 0 & \ldots & 0
\end{array}\right]+I \\
C & =\left[\begin{array}{llll}
1 & 0 & \ldots & 0
\end{array}\right]
\end{aligned}
$$

where $a_{1}, a_{2}, \ldots, a_{n}$ are some unknown parameters to be found. It is important to note that a variant of the observability canonical form is considered here for the pair $(A, C)$, with the difference that the matrix $A$ has an extra term $I$ whose role will become clear later in this work. For the sake of the system identification, define a cost function as follows:

$$
g(\mathbf{y}, \hat{\mathbf{y}}, \hat{\mathbf{u}} ; A, B, d, x[0]):=\sum_{k=0}^{\tau}\|y[k]-\hat{y}[k]\|^{2}
$$

where $\|\cdot\|$ denotes the matrix 2-norm operator and $\mathbf{y}:=$ $\{y[k]\}_{k=0}^{\tau}$. Note that this cost function measures the discrepancy between the experimental data $\hat{\mathbf{y}}$ and the system's output $\mathbf{y}$ when the input $\hat{\mathbf{u}}$ is applied to the system. The goal is to minimize the cost function $g(\mathbf{y}, \hat{\mathbf{y}}, \hat{\mathbf{u}} ; A, B, d, x[0])$ for the parameters $A, B, d, x[0]$. To this end, one can write:

$$
\begin{aligned}
& {\left[\begin{array}{c}
y[0] \\
y[1] \\
y[2] \\
\vdots \\
y[\tau]
\end{array}\right]=\left[\begin{array}{c}
C \\
C A \\
C A^{2} \\
\vdots \\
C A^{\tau}
\end{array}\right] x[0]+\left[\begin{array}{c}
1 \\
1 \\
1 \\
\vdots \\
1
\end{array}\right] d} \\
& +\left[\begin{array}{cccc}
0 & 0 & \cdots & 0 \\
C B & 0 & \cdots & 0 \\
C A B & C B & \cdots & 0 \\
\vdots & \vdots & \ddots & \vdots \\
C A^{\tau-1} B & C A^{\tau-2} B & \cdots & C B
\end{array}\right]\left[\begin{array}{c}
\hat{u}[0] \\
\hat{u}[1] \\
\hat{u}[2] \\
\vdots \\
\hat{u}[\tau-1]
\end{array}\right]
\end{aligned}
$$

It can be concluded from this equality that the minimization of $g(\mathbf{y}, \hat{\mathbf{y}}, \hat{\mathbf{u}} ; A, B, d, x[0])$ may not be a convex problem due to the existence of the product term $A B$. In what follows, a local search method will be proposed, which will be later shown to work satisfactorily for the physiology application. The key idea is that the minimization of $g(\mathbf{y}, \hat{\mathbf{y}}, \hat{\mathbf{u}} ; A, B, d, x[0])$ amounts to a simple least-square problem provided the matrix $A$ is known (in light of the above relation). Hence, the following descent algorithm can be used to find a sub-optimal set of parameters $A, B, d, x[0]$.

Algorithm 1:

- Step 1) Pick an initial Schur matrix $A^{(0)} \in \Re^{n \times n}$ in the form of (2a), and set $i=1$.

- Step 2) Solve a least-squares problem to minimize the cost function $g\left(\mathbf{y}, \hat{\mathbf{y}}, \hat{\mathbf{u}} ; A^{(i-1)}, B, d, x[0]\right)$. Denote the globally optimal values of $B, d$ and $x[0]$ with $B^{(i)}, d^{(i)}$ and $x[0]^{(i)}$, respectively.

- Step 3) Use a local search algorithm to minimize $g\left(\mathbf{y}, \hat{\mathbf{y}}, \hat{\mathbf{u}} ; A, B^{(i)}, d^{(i)}, x[0]^{(i)}\right)$ for a Schur matrix $A$ in 
the form of (2a). Denote a locally optimal value of $A$ with $A^{(i)}$.

- Step 4) If $\left\|A^{(i)}-A^{(i-1)}\right\|+\left\|B^{(i)}-B^{(i-1)}\right\|+\| d^{(i)}-$ $d^{(i-1)}\|+\| x[0]^{(i)}-x[0]^{(i-1)} \|>\varepsilon$ for a given positive tolerance $\varepsilon$, increase $i$ by 1 and then jump to Step 2 .

- Step 5) The system (1) with the parameters $A=A^{(i)}$, $B=B^{(i)}, d=d^{(i)}$ and $x[0]=x[0]^{(i)}$ is a sub-optimal SISO model for the input-output data sets $\hat{\mathbf{u}}$ and $\hat{\mathbf{y}}$.

\section{B. Algorithm for nonlinear SISO systems}

Since the unknown real system associated with the inputoutput data sets $\hat{\mathbf{u}}$ and $\hat{\mathbf{y}}$ may be a nonlinear system, finding the best linear model fitting the data can be potentially too conservative. We consider the class of Hammerstein systems, where each system is composed of a static nonlinear part on the input followed by an LTI model. No result has been reported in the literature to date about the denseness of this class in the space of nonlinear fading-memory systems. Hence, the best Hammerstein model for fitting the data sets $\hat{\mathbf{u}}$ and $\hat{\mathbf{y}}$ may not be able to approximate the real-world system satisfactorily. In what follows, we will discuss how to modify Algorithm 1 to find a locally optimal Hammerstein model. It will be later verified on real experimental data that the physiological responses to exercise can be modeled by Hammerstein systems satisfactorily.

Assume that the unknown SISO system being found for the input-output data sets $\hat{\mathbf{u}}$ and $\hat{\mathbf{y}}$ is required to be of the form

$$
\begin{aligned}
& x[k+1]=A x[k]+f(u[k]) \\
& y[k]=C x[k]+d, \quad k=0,1,2, \ldots
\end{aligned}
$$

as opposed to the affine form (1), where $f(\cdot): \Re \rightarrow \Re^{n}$ is an unknown function to be identified. The space of the unknown parameters of this system is infinite dimensional (due to the presence of $f(u)$ ), which is an obstacle to the underlying system identification problem. To alleviate this issue, one can search for the best $f(u)$ that is representable as a linear combination of a set of pre-specified basis functions. Given the basis functions $f_{1}(u), f_{2}(u), \ldots, f_{\mu}(u)$, we write $f(u)$ as $B \tilde{f}(u)$, where

$$
\tilde{f}(u)=\left[\begin{array}{llll}
f_{1}(u) & f_{2}(u) & \cdots & f_{\mu}(u)
\end{array}\right]^{T}
$$

and $B \in \Re^{n \times \mu}$ is the matrix of unknown coefficients. The data fitting problem now amounts to finding the best model in the form of

$$
\begin{aligned}
& x[k+1]=A x[k]+B u \\
& y[k]=c x[k]+d, \quad k=0,1,2, \ldots
\end{aligned}
$$

for the input-output sequences $\{\tilde{f}(\hat{u}[k])\}_{k=0}^{\tau}$ and $\{\hat{y}[k]\}_{k=0}^{\tau}$. One can adopt Algorithm 1 to find a sub-optimal set of the parameters $A, B, d, x[0]$.

\section{Algorithm for universal nonlinear SISO systems}

Given a natural number $p$, consider $2 p$ input-output sequences $\hat{\mathbf{u}}_{j}:=\left\{\hat{u}_{j}[k]\right\}_{k=0}^{\tau}$ and $\hat{\mathbf{y}}_{j}:=\left\{\hat{y}_{j}[k]\right\}_{k=0}^{\tau}, j=$ $1,2, \ldots, p$. The objective of this part is to find a matrix $A \in \Re^{n \times n}$, a function $f: \Re \rightarrow \Re^{n}$, scalars $d_{1}, d_{2}, . ., d_{p}$ and vectors $x_{1}[0], x_{2}[0], \ldots, x_{p}[0] \in \Re^{n}$ for which the output of the system

$$
\begin{aligned}
& x[k+1]=A x[k]+f(u[k]) \\
& y[k]=c x[k]+d_{j}, \quad k=0,1,2, \ldots
\end{aligned}
$$

with the initial state $x[0]=x_{j}[0]$ in response to the input $\hat{\mathbf{u}}_{j}$ is as closely as possible to $\hat{\mathbf{y}}_{j}$ over the time period $[0, \tau]$ for every $j \in\{1,2, \ldots, p\}$. We call this system a universal system. The primary application of this problem is in finding a universal model for different athletes doing exercise. To address this problem, the first step is to make the parameters space finite dimensional using the aforementioned basis function technique. Thus, a model in the form of (7) is sought. A variant of Algorithm 1 can now be used for this purpose.

\section{Algorithm 2:}

- Step 1) Select an initial Schur matrix $A^{(0)} \in \Re^{n \times n}$ in the form of 2a), and set $i=1$.

- Step 2) Solve a least-squares problem to minimize the cost function

$$
\sum_{j=1}^{p} g\left(\mathbf{y}, \hat{\mathbf{y}}_{j}, \hat{\mathbf{u}}_{j} ; A^{(i-1)}, B, d_{j}, x_{j}[0]\right)
$$

Denote the globally optimal values of the relevant parameters with $B^{(i)}, d_{j}^{(i)}$ and $x_{j}[0]^{(i)}$, for $j=1,2, \ldots, p$.

- Step 3) Use a local search algorithm (such as a gradient method) to minimize:

$$
\sum_{j=1}^{p} g\left(\mathbf{y}, \hat{\mathbf{y}}_{j}, \hat{\mathbf{u}}_{j} ; A, B^{(i)}, d_{j}^{(i)}, x_{j}[0]^{(i)}\right)
$$

for a Schur matrix $A$ in the form of 2a. Denote a locally optimal value of $A$ with $A^{(i)}$.

- Step 4) If $\left\|A^{(i)}-A^{(i-1)}\right\|+\left\|B^{(i)}-B^{(i-1)}\right\|+$ $\sum_{j=1}^{p}\left\|d_{j}^{(i)}-d_{j}^{(i-1)}\right\|+\sum_{j=1}^{p}\left\|x_{j}[0]^{(i)}-x_{j}[0]^{(i-1)}\right\|>\varepsilon$ for a given positive tolerance $\varepsilon$, increase $i$ by 1 and then jump to Step 2.

- Step 5) The system 8 with the parameters $A=A^{(i)}$, $f(u)=B^{(i)} \tilde{f}(u), d_{j}=d_{j}^{(i)}$ and $x[0]=x_{j}[0]^{(i)}$ is a suboptimal SISO model for the input-output data sets $\hat{\mathbf{u}}_{j}$ and $\hat{\mathbf{y}}_{j}$, for every $j \in\{1,2, \ldots, p\}$.

The analysis provided in [29] can be used to partially justify the convergence of the above algorithm to a global solution under technical conditions.

\section{Algorithm for linear SIMO systems}

Given a natural number $r$, consider an input sequence $\hat{\mathbf{u}}:=$ $\{\hat{u}[k]\}_{k=0}^{\tau}$ and $r$ output sequences $\hat{\mathbf{y}}_{j}:=\left\{\hat{y}_{j}[k]\right\}_{k=0}^{\tau}, j=$ $1,2, \ldots, r$. Define:

$$
\hat{y}[k]=\left[\begin{array}{llll}
\hat{y}_{1}[k] & \hat{y}_{2}[k] & \cdots & \hat{y}_{r}[k]
\end{array}\right]^{T}, \quad k=0,1,2, \ldots
$$

and $\hat{\mathbf{y}}:=\{\hat{y}[k]\}_{k=0}^{\tau}$. The objective is to find a single-input $r$-output system of the form:

$$
\begin{aligned}
& x[k+1]=A x[k]+B u[k] \\
& y[k]=C x[k]+d, \quad k=0,1,2, \ldots
\end{aligned}
$$


such that $\mathbf{y}:=\{y[k]\}_{k=0}^{\tau}$ is as closely as possible to $\hat{\mathbf{y}}$ if the input $\hat{\mathbf{u}}$ is applied to the system. The unknown parameters of the system identification problem are $A \in \Re^{n \times n}, B \in$ $\Re^{n}, C \in \Re^{r \times n}$ and $x[0] \in \Re^{n}$. Algorithms 1 and 2 provided in the preceding subsections were based on the fact that finding the optimal values of the parameters is a least-squares problem if the matrix $A$ is known. However, this idea breaks down for the identification of a SIMO system. More precisely, there does not exist an observable canonical form for a SIMO system to start with. Since the unknown system being identified has a single input, one may realize it in the controllable canonical form. Nonetheless, this does not help because the corresponding optimization problem is not convex in that case even if $A$ is known a priori. To resolve the issue, we find $r$ SISO systems:

$$
\begin{aligned}
& x_{j}[k+1]=A x_{j}[k]+B_{j} u[k] \\
& y_{j}[k]=\left[\begin{array}{cccc}
1 & 0 & \cdots & 0
\end{array}\right] x_{j}[k]+d_{j}, \quad k=1,2, \ldots
\end{aligned}
$$

(where $j=1,2, \ldots, r$ ) with the parameters $A \in \Re^{n \times n}$, $B_{1}, \ldots, B_{r} \in \Re^{n}, d_{1}, \ldots, d_{r} \in \Re$ and $x_{1}[0], \ldots, x_{r}[0] \in \Re^{n}$ such that the following cost function is locally minimized:

$$
\sum_{j=1}^{r} g\left(\mathbf{y}_{j}, \hat{\mathbf{y}}_{j}, \hat{\mathbf{u}} ; A, B_{j}, d_{j}, x_{j}[0]\right)
$$

where $\mathbf{y}_{j}:=\left\{y_{j}[k]\right\}_{k=0}^{\tau}$. Algorithm 2 can be easily modified to find a sub-optimal solution of this problem. Thus, $r$ SISO systems can be obtained for the $r$ individual input-output data sets $\mathbf{u}$ and $\hat{\mathbf{y}}_{\mathbf{j}}, j=1,2, \ldots, r$, so that they all have the same matrix $A$. The next step is to combine these SISO systems to obtain a single-input $r$-output system corresponding to the input-output data sets $\mathbf{u}$ and $\hat{\mathbf{y}}$. To this end, we form a parallel connection of these $r$ systems as follows:

$$
\begin{aligned}
& \tilde{x}[k+1]=\tilde{A} \tilde{x}[k]+\tilde{B} u[k] \\
& y[k]=\tilde{C} \tilde{x}[k]+d
\end{aligned}
$$

where:

- $\tilde{A} \in \Re^{r n \times r n}$ is a block diagonal matrix with the $(j, j)^{\text {th }}$ block $A$ for every $j \in\{1,2, \ldots, r\}$.

- $\tilde{B} \in \Re^{n r}$ is a column vector obtained from $B_{1}, B_{2}, \ldots, B_{r}$ by stacking them up.

- $\tilde{C} \in \Re^{r \times r n}$ is a block diagonal matrix with the $(j, j)^{\text {th }}$ block entry $\left[\begin{array}{llll}1 & 0 & \cdots & 0\end{array}\right]$ for every $j \in\{1,2, \ldots, r\}$.

- $\tilde{d} \in \Re^{r}$ is a column vector obtained from $d_{1}, d_{2}, \ldots, d_{r}$ by stacking them up.

The obtained system (15) is a near-optimal model for the data sets $\mathbf{u}$ and $\hat{\mathbf{y}}$, but its order is $n r$ rather than the desired value $n$. Due to the particular structure of the matrix $\tilde{A}$, it can be observed that the system $(\tilde{A}, \tilde{b}, \tilde{C})$ is uncontrollable and indeed it has a realization $(A, B, C)$ where $A \in \Re^{n \times n}, B \in \Re^{n}$ and $C \in \Re^{r \times n}$.

So far, a single-input $r$-output system of order $n$ is obtained whose transfer function from its input to its $j^{\text {th }}$ output is equal to the transfer function of the $j^{\text {th }}$ SISO system 13 found earlier, for every $j \in\{1,2, \ldots, r\}$. The only issue that may cause a problem is that the $r$ separate initial states $x_{1}[0], x_{2}[0], \ldots, x_{r}[0]$ obtained by solving $r$ SISO system identification problems cannot always be incorporated into a single initial state for the reduced-order model.

\section{MathematicAl MOdel OF PHYSIOLOGICAL RESPONSE}

This section obtains empirical mathematical models for the physiological responses to exercise. The algorithms proposed in the previous section will be deployed to find satisfactory models from the data gathered from four different experiments of running on treadmill. The input watts corresponding to these experiments are given in Figure 1 The first goal is to identify a model of oxygen variability $\left(\dot{V}_{\mathrm{O} 2}\right)$ with respect to the workload intensity $(W)$. Algorithm 1 is used to study the possibility of the existence of an accurate first-order linear model for each of these experiments. Figure 2 shows the output of the first-order linear model of $\dot{V}_{\mathrm{O} 2}$ for Experiment 1. It can be observed that the model obtained by the proposed heuristic method fits the data very well. An important implication of this result is that the $\dot{V}_{O 2}$ response to $W$ is governed by a simple first-order differential equation (this phenomenon was observed for other experiments as well).

The $\dot{V}_{O 2}$ signals for Experiments 1 to 4 are very similar in nature. Hence, one may speculate that there exists a universal model for the response of $\dot{V}_{O 2}$ to $W$. To study this conjecture, we first use Algorithm 1 to find three separate first-order linear models corresponding to $\dot{V}_{O 2}$ for Experiments 1 to 3 and then deploy Algorithm 2 to obtain a single universal first-order linear model. The corresponding plots are given in Figure 3. which clearly demonstrate that a simple system obtained by Algorithm 2 for $\dot{V}_{O 2}$ works satisfactorily for different experiments.

In contrast to the $\dot{V}_{O 2}$ signal, the experimental data suggests that the ventilation variability $\left(\dot{V}_{V E}\right)$ is more complex and probably more individual. For each of the underlying experiments, Algorithm 1 failed to find a satisfactory firstorder linear system modeling the $\dot{V}_{V E}$ response to $W$. This can be due to the nonexistence of such a model. Nonetheless, an acceptable second-order linear model can always be found for Experiments 1 to 4. For Experiment 1, the model's output is compared to the experimental data in Figure 4. The plots clearly show that the response of $\dot{V}_{V E}$ to $W$ can be modeled via a second-order linear system.

The next quantity of interest is the difference between $\mathrm{CO} 2$ production and $\mathrm{O} 2$ consumption, i.e., the $\mathrm{CO} 2-\mathrm{O} 2$ variability. Our study on a number of different data sets reveals that $\dot{V}_{C O 2-O 2}$ is far more complex than $\dot{V}_{O 2}, \dot{V}_{V E}, \dot{V}_{H R}$ and, therefore, its governing model is unlikely to be a low-order linear system. The best second-order linear model fitting the $\dot{V}_{\mathrm{CO} 2-\mathrm{O} 2}$ data is compared with the real data in Figure 5 for Experiment 1 to demonstrate the poor performance of a linear system. To find a nonlinear model for the same experiment, define the following basis functions:

$$
f_{i}(u)=\max \left(0, u-\tau_{i}\right), \quad i=1,2,3
$$

where:

$$
\tau_{1}=0, \quad \tau_{2}=220, \quad \tau_{3}=290
$$




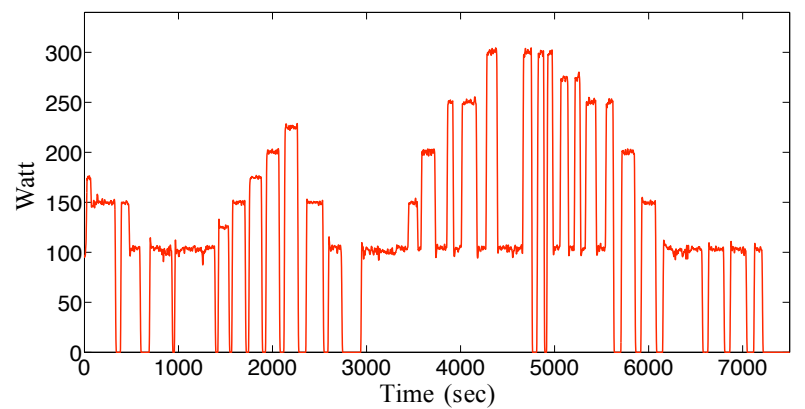

(a)

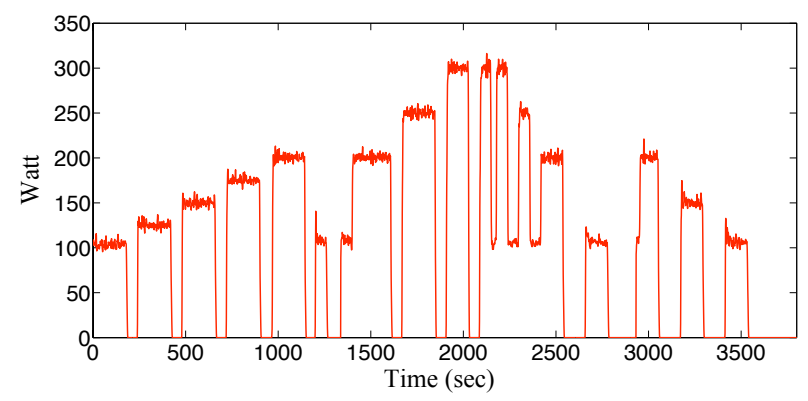

(c)

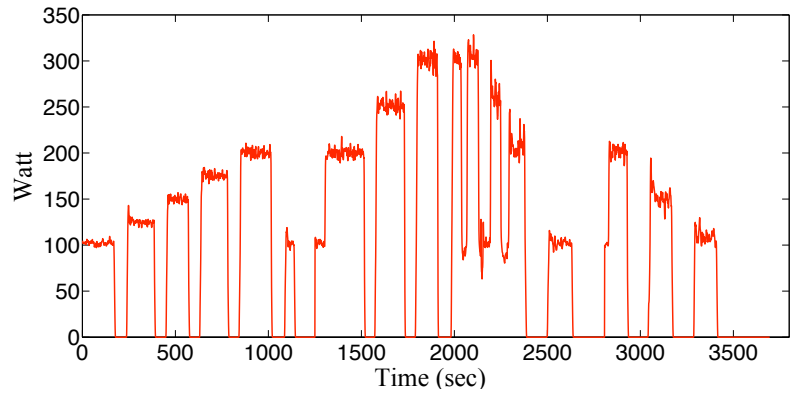

(b)

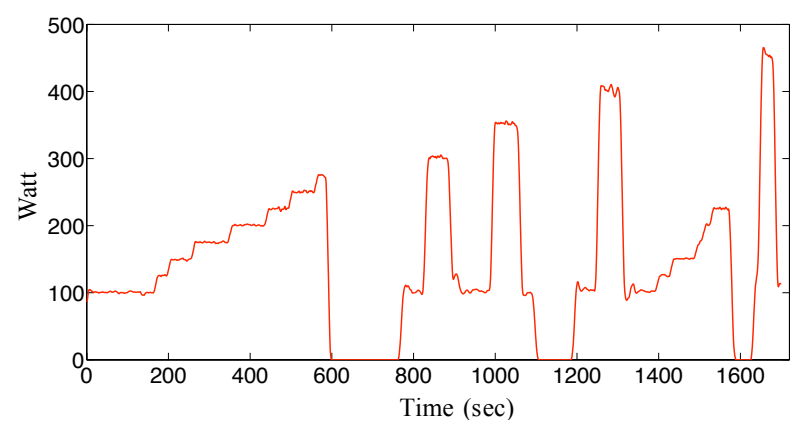

(d)

Fig. 1: (a)-(d): The input signals for Experiments 1-4.

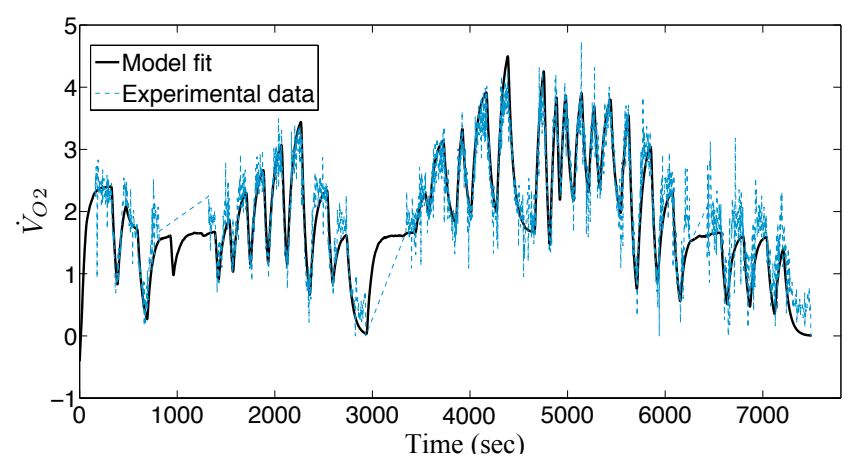

Fig. 2: The output of the first-order linear model corresponding to $\dot{V}_{O 2}$ for Experiment 1 .

Using only three simple basis functions as given above, the method proposed here is used to find a second-order nonlinear system modeling the $\dot{V}_{\mathrm{CO} 2-\mathrm{O} 2}$ response to $W$. The corresponding plots are given in Figure 6 to show how accurately the fits describe the data. The signals $g_{1}(u)$ and $g_{2}(u)$ in the figure are the first and second entries of the nonlinear vector function $f(u)$. Note that these nonlinear functions are very simple and a low-order system with mild nonlinearities can model the underlying response satisfactorily. We noticed that a superior fitting can be attained by considering more basis functions, which leads to a complicated nonlinearity on the input.

It is desirable to find an integrated single-input four-output system modeling all responses $\dot{V}_{O 2}, \dot{V}_{C O 2}, \dot{V}_{V E}$, and $\dot{V}_{H R}$ to $W$. By applying the method proposed in Subsection $D$ to
Experiment 1, it can be observed that there exists a secondorder linear model capturing these four individual responses. The corresponding plots are given in Figure 7 to illustrate how well a low-order linear model can predict four different quantities $\dot{V}_{O 2}, \dot{V}_{C O 2}, \dot{V}_{V E}$ and $\dot{V}_{H R}$ from $W$. Similarly, there exist integrated linear single-input four-output systems of order 2 for other experiments, and Figure 8 shows the results obtained for experiment 4.

As stated earlier, the $\dot{V}_{H R}$ response to $W$ can be modeled by a low-order linear system, whereas the $\dot{V}_{\mathrm{CO} 2-\mathrm{O} 2}$ response to $W$ requires a nonlinear system. The question arises as to whether the models for $\dot{V}_{\mathrm{HR}}$ and $\dot{V}_{\mathrm{CO} 2-\mathrm{O} 2}$ can be governed by the same dynamics (i.e., an identical $A$ matrix). Algorithm 2 is used for Experiment 4 to find the best thirdorder nonlinear models for $\dot{V}_{\mathrm{HR}}$ and $\dot{V}_{\mathrm{CO} 2-\mathrm{O} 2}$ when their dynamics are forced to be identical. The models' signals are compared with experimental signals in Figure 9 The functions $g_{1}(u), g_{2}(u), g_{3}(u)$ and $\bar{g}_{1}(u), \bar{g}_{2}(u), \bar{g}_{3}(u)$ in the figure denote the input nonlinearities of the models for $\dot{V}_{H R}$ and $\dot{V}_{\mathrm{CO} 2-\mathrm{O} 2}$, respectively.

Remark 1: It is expected that a physiological model corresponding to each of the signals $\dot{V}_{H R}, \dot{V}_{O 2}, \dot{V}_{C O 2}$, and $\dot{V}_{V E}$ has a non-decreasing input function $f(u)$. This implies that the coefficient matrix $B$ being found should be constrained to have only nonnegative entries. This constraint can be easily incorporated into Algorithm 1 or Algorithm 2 by imposing to solve a nonnegative least-squares problem as opposed to a standard least-squares problem. The corresponding simulations given above are all obtained based on imposing a nonnegativity constraint on $B$.

Remark 2: It can be observed in Figures 2, 4, 5, and 7 that 

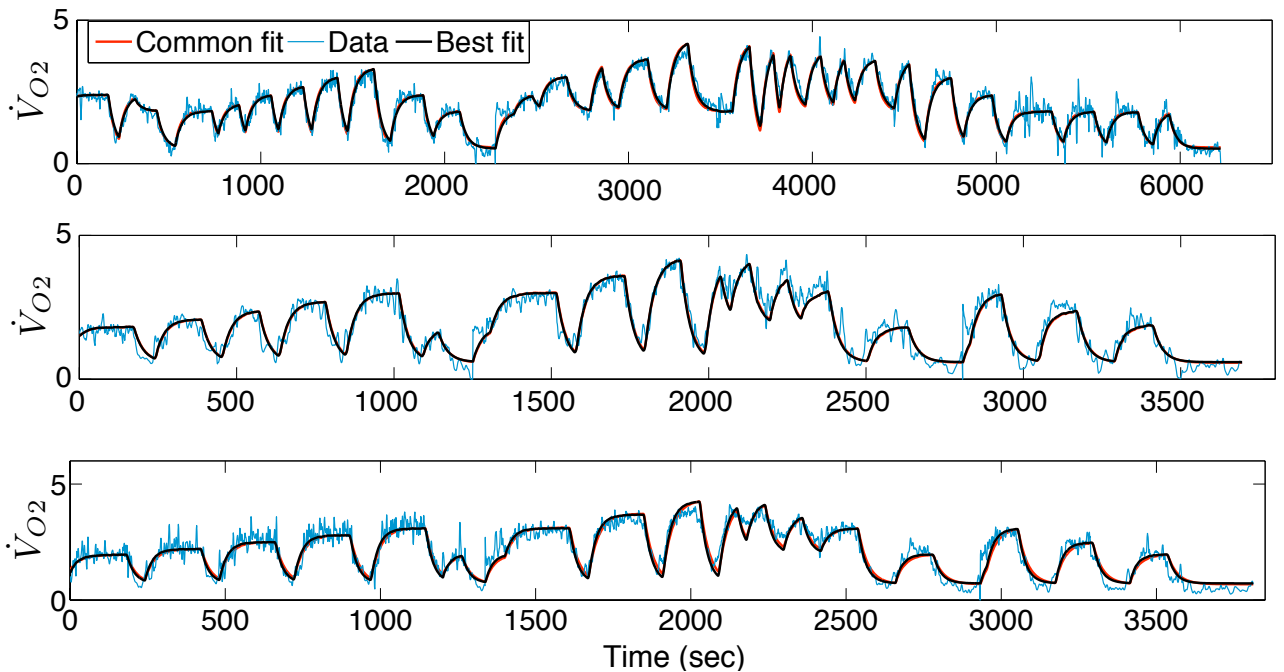

Fig. 3: A universal first-order linear model corresponding to $\dot{V}_{O 2}$ for Experiments 1,2 and 3 is compared with three individual first-order linear models for these experiments.

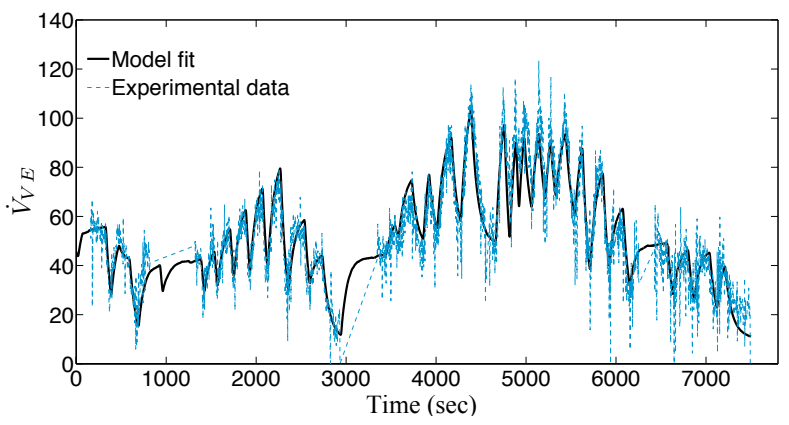

Fig. 4: The output of the second-order linear model corresponding to $\dot{V}_{V E}$ for Experiment 1.

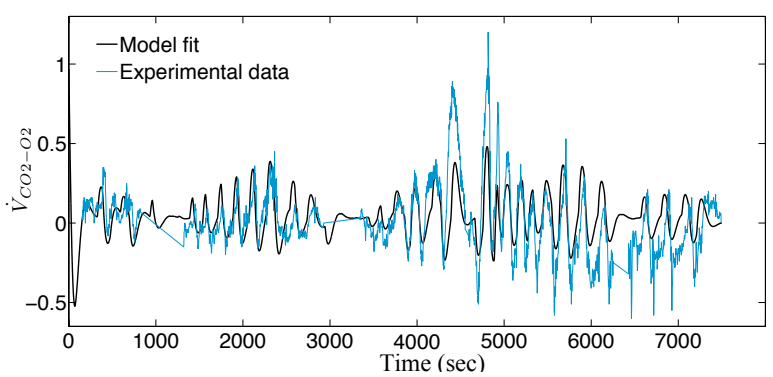

Fig. 5: The output of the second-order linear model corresponding to $\dot{V}_{\mathrm{CO} 2-\mathrm{O} 2}$ for Experiment 1.

the experimental data is simply a line over three distinct time intervals. The reason is that the data could not be measured in those intervals and therefore a simple line interpolation method was used to predict the missing data. Hence, the model's output may not fit the experimental data very well on these intervals as the unmeasured real data is not expected to be a line.

Remark 3: One may use an off-the-shelf nonlinear solver to find an empirical model based on the existing system identification techniques. However, we have observed that the resulting estimation problems based on our data are highly nonlinear that a generic local search algorithm could easily become trapped in a local solution such that its corresponding model does not fit the data well. Although due to space restrictions the results of those unsatisfactory models are not presented here, the models found using our customized numerical algorithms fit the data very well. The main contribution of this work is the findings and observations made on the obtained models.

\section{CONCLUSions}

This work develops empirical mathematical models for the physiological responses to exercise. In particular, the variability of heart rate $(H R)$, ventilation $(V E)$, oxygen $(O 2)$ and carbon dioxide $(C O 2)$ to workload intensity $(W)$ is studied. Based on simulations performed on different experimental data, we observe that: i) the response of each of the signals $H R, V E, O 2$ and $C O 2$ to $W$ can be modeled by either a first- or a second-order linear system; ii) there exists a single (universal) first-order linear model for the $\mathrm{O} 2$ variability of different individuals; iii) the effect of $W$ on all of the signals $H R, V E, O 2$ and $C O 2$ can be captured by a single-input fouroutput second-order linear model. Besides, this study shows that the response from $\mathrm{W}$ to $\mathrm{O} 2-\mathrm{CO} 2$ cannot be modeled by a first- or second-order system unless mild nonlinearities are incorporated in the input of the system.

\section{REFERENCES}

[1] R. Pintelon and J. Schoukens, System identification: a frequency domain approach. John Wiley \& Sons, 2012.

[2] T. Katayama, Subspace methods for system identification. Springer Science \& Business Media, 2006.

[3] I. Uyanik, U. Saranli, M. M. Ankarali, N. J. Cowan, and O. Morgul, "Frequency-domain subspace identification of linear time periodic (LTP) systems," IEEE Transactions on Automatic Control, 2018.

[4] S. Fattahi and S. Sojoudi, "Data-driven sparse system identification," arXiv preprint arXiv:1803.07753, 2018. 


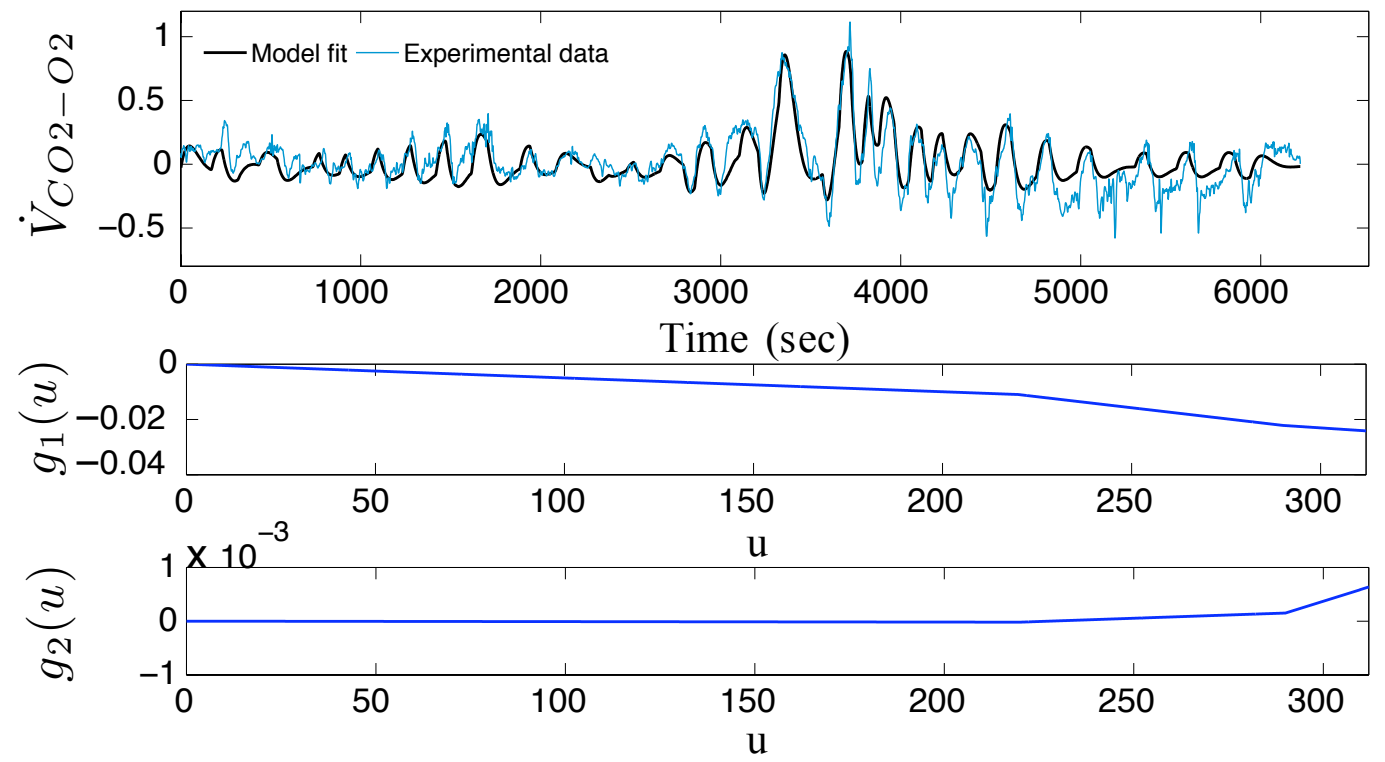

Fig. 6: The relevant signals of the second-order nonlinear model corresponding to $\dot{V}_{\mathrm{CO} 2-\mathrm{O} 2}$ for Experiment 1 .
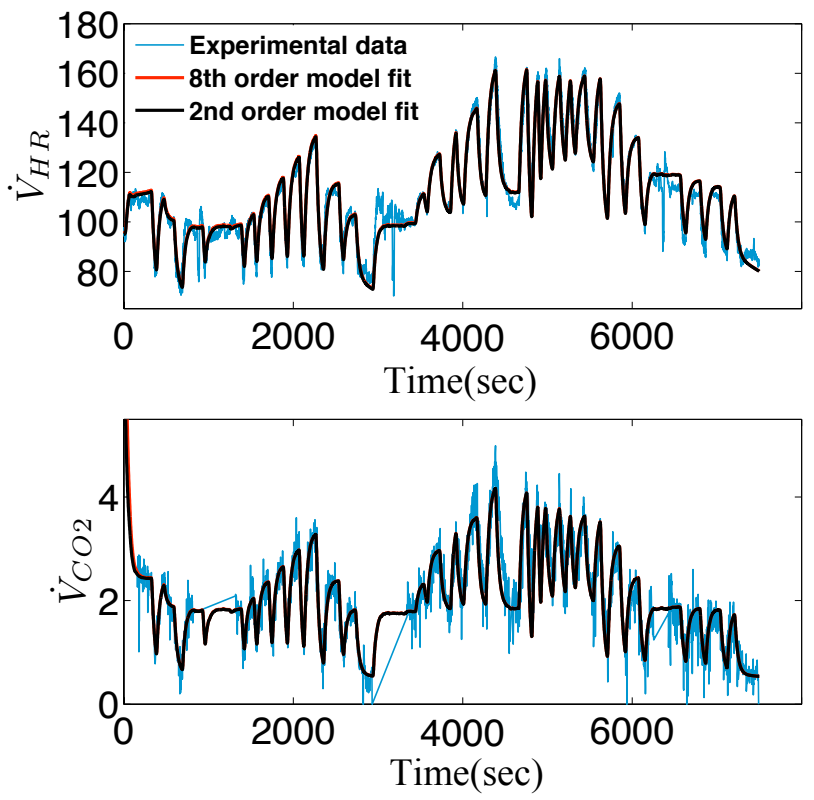
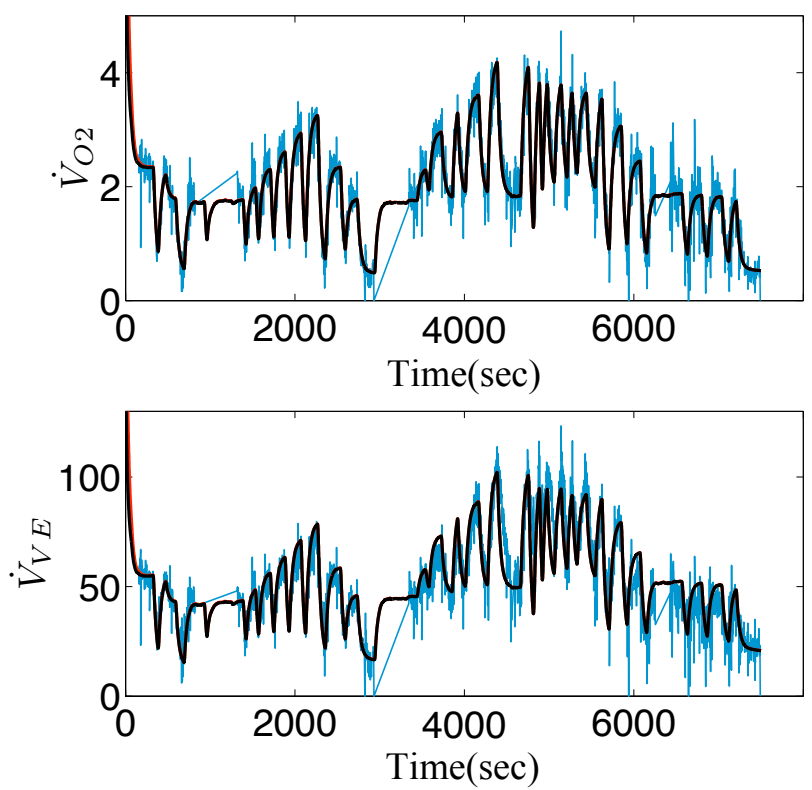

Fig. 7: The outputs of the integrated second-order linear model for Experiment 1.

[5] O. Nelles, Nonlinear system identification: from classical approaches to neural networks and fuzzy models. Springer Science \& Business Media, 2013.

[6] J. Sjöberg, Q. Zhang, L. Ljung, A. Benveniste, B. Delyon, P.-Y. Glorennec, H. Hjalmarsson, and A. Juditsky, "Nonlinear black-box modeling in system identification: a unified overview," Automatica, vol. 31, no. 12, pp. 1691-1724, 1995.

[7] W. J. Rugh, Nonlinear system theory. Johns Hopkins University Press Baltimore, 1981.

[8] K. Li, J.-X. Peng, and G. W. Irwin, "A fast nonlinear model identification method," IEEE Transactions on Automatic Control, vol. 50, no. 8, pp. 1211-1216, 2005.

[9] D. T. Westwick and R. E. Kearney, "Separable least squares identification of nonlinear hammerstein models: Application to stretch reflex dynamics," Annals of Biomedical Engineering, vol. 29, no. 8, pp. 707718, 2001.

[10] I. W. Hunter and M. J. Korenberg, "The identification of nonlinear bi- ological systems: Wiener and hammerstein cascade models," Biological cybernetics, vol. 55, no. 2-3, pp. 135-144, 1986.

[11] E.-W. Bai, Z. Cai, S. Dudley-Javorosk, and R. K. Shields, "Identification of a modified wiener-hammerstein system and its application in electrically stimulated paralyzed skeletal muscle modeling," Automatica, vol. 45, no. 3, pp. 736-743, 2009.

[12] I. Goethals, K. Pelckmans, J. A. Suykens, and B. De Moor, "Subspace identification of hammerstein systems using least squares support vector machines," IEEE Transactions on Automatic Control, vol. 50, no. 10, pp. 1509-1519, 2005.

[13] R. S. Risuleo, G. Bottegal, and H. Hjalmarsson, "A kernel-based approach to hammerstein system identification," IFAC-PapersOnLine, vol. 48, no. 28, pp. 1011-1016, 2015.

[14] M. A. Dahleh, "Asymptotic worst-case identification with bounded noise," in The Modeling of Uncertainty in Control Systems. Springer, 1994, pp. 157-170.

[15] A. Julius, M. Zavlanos, S. Boyd, and G. J. Pappas, "Genetic network 

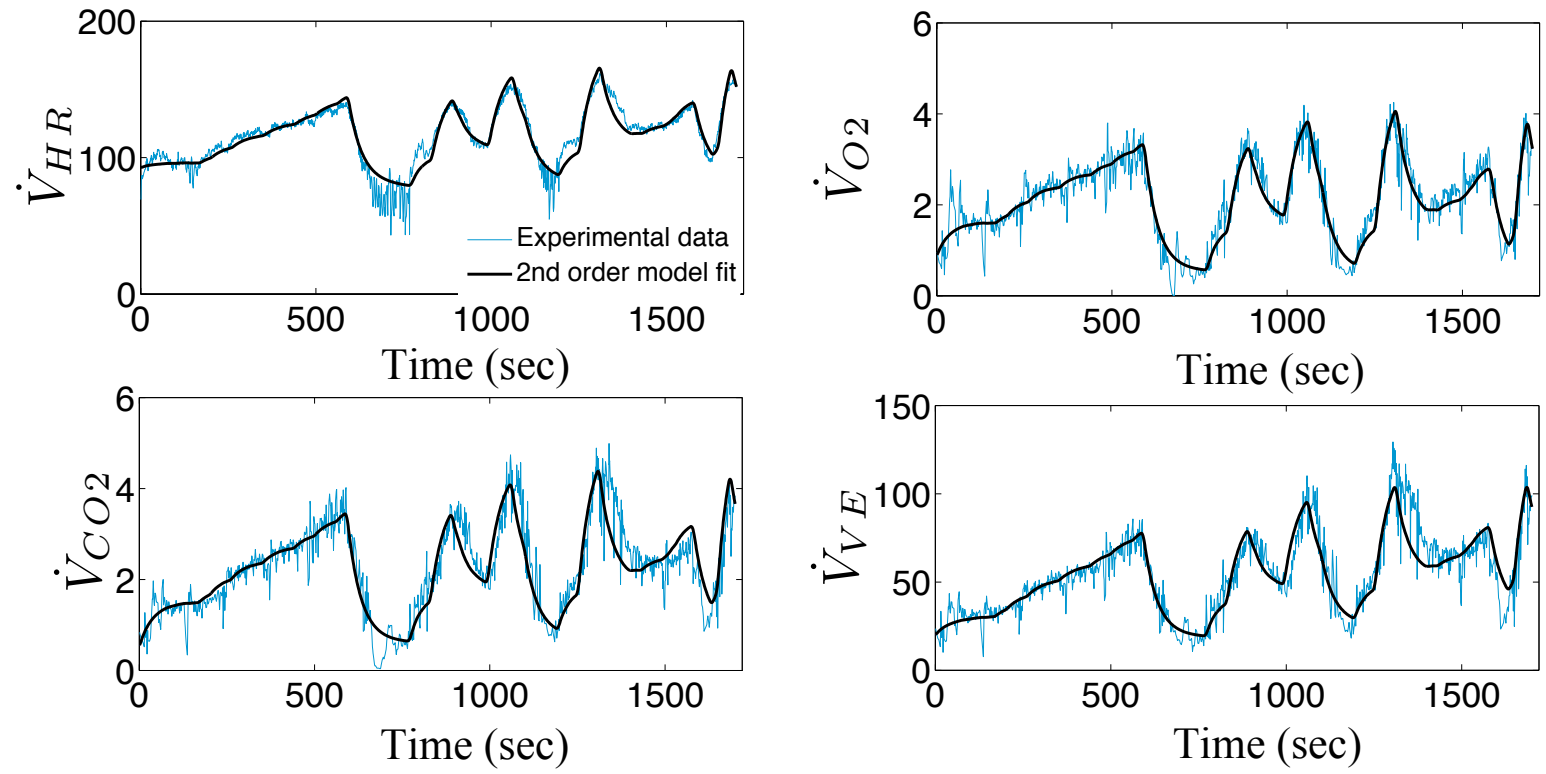

Fig. 8: The outputs of the integrated second-order linear model for Experiment 4.
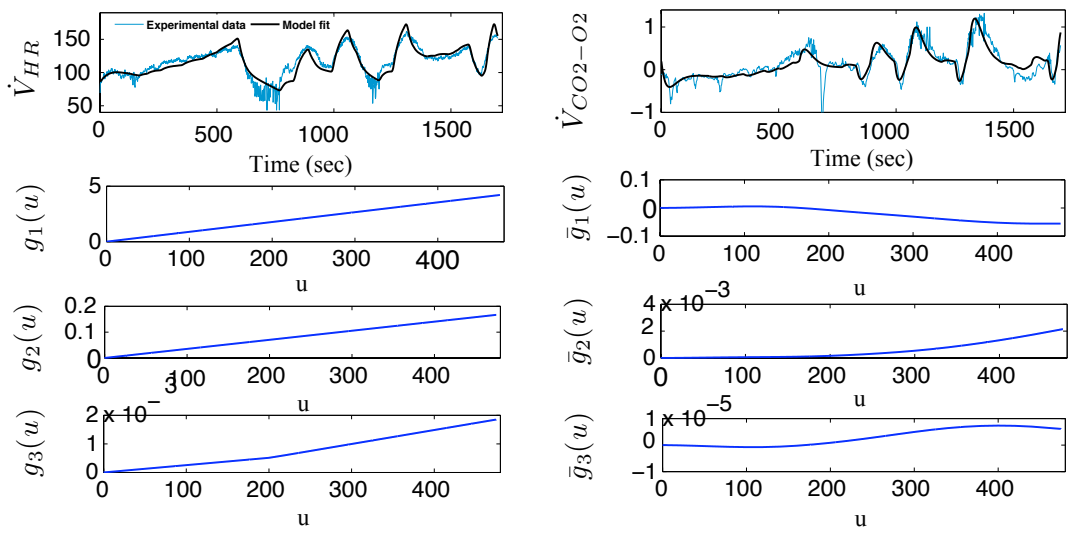

Fig. 9: Different signals for the third-order nonlinear models corresponding to $\dot{V}_{\mathrm{HR}}$ and $\dot{V}_{\mathrm{CO} 2-\mathrm{O} 2}$ for Experiment 4.

identification using convex programming," IET systems biology, vol. 3, no. 3, pp. 155-166, 2009.

[16] M. M. Zavlanos, A. A. Julius, S. P. Boyd, and G. J. Pappas, "Inferring stable genetic networks from steady-state data," Automatica, vol. 47, no. 6, pp. 1113-1122, 2011.

[17] M. J. Korenberg, "Applications of nonlinear system identification in molecular biology," in Engineering in Medicine and Biology Society, 2006. EMBS'06. 28th Annual International Conference of the IEEE. IEEE, 2006, pp. 256-259.

[18] X. Xiao, T. J. Mullen, and R. Mukkamala, "System identification: a multi-signal approach for probing neural cardiovascular regulation," Physiological measurement, vol. 26, no. 3, p. R41, 2005.

[19] H. Wang, K. Ju, and K. H. Chon, "Closed-loop nonlinear system identification via the vector optimal parameter search algorithm: application to heart rate baroreflex control," Medical engineering \& physics, vol. 29, no. 4, pp. 505-515, 2007.

[20] T. M. Cheng, A. V. Savkin, B. G. Celler, S. W. Su, L. Wang et al., "Nonlinear modeling and control of human heart rate response during exercise with various work load intensities," IEEE Transactions on biomedical engineering, vol. 55, no. 11, pp. 2499-2508, 2008.

[21] T. J. Barstow and P. A. Molé, "Linear and nonlinear characteristics of oxygen uptake kinetics during heavy exercise," Journal of Applied Physiology, vol. 71, no. 6, pp. 2099-2106, 1991.

[22] K. J. Hunt, B. Ajayi, H. Gollee, and L. Jamieson, "Feedback control of oxygen uptake during treadmill exercise," IEEE Transactions on Control
Systems Technology, vol. 16, no. 4, pp. 624-635, 2008.

[23] N. Li, J. Cruz, C. S. Chien, S. Sojoudi, B. Recht, D. Stone, M. Csete, D. Bahmiller, and J. C. Doyle, "Robust efficiency and actuator saturation explain healthy heart rate control and variability," Proceedings of the National Academy of Sciences, vol. 111, no. 33, pp. E3476-E3485, 2014.

[24] R. Ge, J. D. Lee, and T. Ma, "Matrix completion has no spurious local minimum," in Advances in Neural Information Processing Systems (NIPS), 2016.

[25] S. Bhojanapalli, B. Neyshabur, and N. Srebro, "Global optimality of local search for low rank matrix recovery," in Advances in Neural Information Processing Systems (NIPS), 2016.

[26] Y. Chen, Y. Chi, J. Fan, and C. Ma, "Gradient descent with random initialization: Fast global convergence for nonconvex phase retrieval," arXiv preprint arXiv:1803.07726, 2018.

[27] R. Y. Zhang, C. Josz, S. Sojoudi, and J. Lavaei, "How much restricted isometry is needed in nonconvex matrix recovery?" arXiv preprint arXiv:1805.10251, 2018.

[28] C. Josz, Y. Ouyang, R. Zhang, J. Lavaei, and S. Sojoudi, "A theory on the absence of spurious optimality," arXiv preprint arXiv:1805.08204, 2018.

[29] M. Hardt, T. Ma, and B. Recht, "Gradient descent learns linear dynamical systems," arXiv preprint arXiv:1609.05191, 2016. 